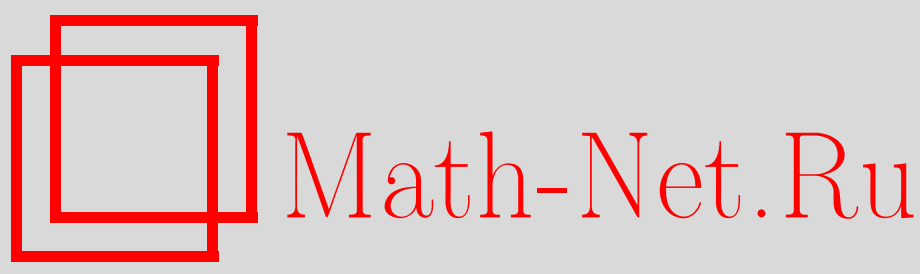

М. М. Гриненко, О двойном конусе над поверхностью Веронезе, Изв. РАН. Сер. матем., 2003, том 67, выпуск 3, 5-22

DOI: https://doi.org/10.4213/im433

Использование Общероссийского математического портала Math-Net.Ru подразумевает, что вы прочитали и согласны с пользовательским соглашением

http://www . mathnet.ru/rus/agreement

Параметры загрузки:

IP : 54.224 .135 .184

26 апреля 2023 г., 14:56:41 
УДК 512.6

\title{
М.М. Гриненко
}

\section{О двойном конусе над поверхностью Веронезе}

\author{
К 80-летию Игоря Ростиславовича Шафаревича
}

\begin{abstract}
Изучается бирациональная геометрия трехмерного многообразия Фано, являющегося двойным накрытием конуса над поверхностью Веронезе с ветвлением в неособом сечении кубикой. В частности, обсуждается множество различных структур Мори, а также возможные типы максимальных особенностей линейных систем.

Библиография: 10 наименований.
\end{abstract}

\section{§1. Предварительные замечания}

Пусть $X_{4} \subset \mathbb{P}^{5}$ - поверхность Веронезе (т.е. плоскость, вложенная в $\mathbb{P}^{5}$ при помоши полной линейной системы коник), $Q \subset \mathbb{P}^{6}-$ конус над ней с вершиной в точке $Q_{0}, R \subset \mathbb{P}^{6}$ - кубическая гиперповерхность такая, что сечение $R_{Q}=R \cap Q$ неособо и не проходит через $Q_{0}$. Легко видеть, что сушествует двойное накрытие $\varphi: V \stackrel{2: 1}{\rightarrow} Q$ с ветвлением в дивизоре $R_{Q}, V$ является многообразием Фано индекса 2 основной серии с $\left(-K_{V}\right)^{3}=8$. Точнее,

$$
\operatorname{Pic}(V)=\mathbb{Z} H
$$

причем $K_{V} \sim-2 H, H^{3}=1$. Поверхности класса $H$ образуют двумерное семейство (т.е. $H^{0}(V, H)=3$ ) поверхностей дель Пецщо степени 1. Точка $P \in V$, лежашая над вершиной конуса $Q_{0} \in Q$, является единственной базисной точкой этого семейства. Класс $l$ кривых на $V$, являюшихся прообразами образуюших конуса, порождает группу одномерных циклов. Легко видеть, что все эти кривые (а они составляют двумерное семейство $\mathscr{L}$, параметризованное $X_{4}$ ) неприводимы. Общий элемент семейства является неособой эллиптической кривой, а одномерное подсемейство вырождений представлено рациональными кривыми с нодальной либо каспидальной особенностью. Кривая из семейства $\mathscr{L}$ трансверсально пересекает в точке $P$ любую поверхность класса $H$, не содержащую ее, и это - единственная точка их пересечения. Очевидно, $l \circ H=1$.

Напомним, что трехмерное нормальное проективное многообразие $U$ называется расслоением Мори относительно морфизма $\rho: U \rightarrow S$ на нормальное многообразие $S$ размерности, строго меньшей, чем размерность $U$, если $U$ имеет $\mathbb{Q}$-факториальные терминальные особенности и $\rho$ является экстремальным стягиванием

Работа выполнена при финансовой поддержке Фонда содействия отечественной науке, Российского фонда фундаментальных исследований (гранты № 02-01-00441 и№ 00-15-96085), NWO-RFBR № 047-008-005, INTAS № 00-0269 и № 00-0259. 
расслоенного типа. Говорят также, что расслоения Мори $\rho: U \rightarrow S$ и $\rho^{\prime}: U^{\prime} \rightarrow S^{\prime}$ бирачиональны над базой, или имеют одну и ту же структуру Мори, если:

1) при $\operatorname{dim} S>0$ существуют бирациональные отображения $\chi: U \rightarrow U^{\prime}$ и $\psi: S \rightarrow S^{\prime}$ такие, что $\rho^{\prime} \circ \chi=\psi \circ \rho$;

2 ) при $\operatorname{dim} S=0$ многообразия $U$ и $U^{\prime}$ бирегулярно изоморфны.

$\mathrm{C}$ точки зрения современного подхода, основная задача бирациональной классификации состоит в описании различных структур Мори для данного многообразия.

Заметим, что в рассматриваемом случае $V$ само является расслоением Мори (со структурой многообразия Фано). Кроме того, существует также “двумерное семейство" расслоений Мори, “параметризованных" $X_{4}$, следующего вида. Кривая $C \in \mathscr{L}$ совпадает с базисным множеством пучка $|H-C|$. Более того, $C$ является полным пересечением любых двух элементов этого пучка. Пусть $V_{C} \rightarrow V-$ раздутие в кривой $C$. Тогда $V_{C}$ является расслоением Мори на поверхности дель Пеццо степени 1 относительно естественной проекции на $\mathbb{P}^{1}$. Собственные прообразы элементов пучка $|H-C|$ будут слоями этой проекции. Очевидно, $V_{C}$ неособо, если неособа $C$. Если $C$ имеет нодальную особенность, то $V_{C}$ имеет простейшую квадратичную особенность в некоторой точке. Наконец, если $C$ имеет каспидальную особенность, то $V_{C}$ имеет двойную точку, которая в подходящих локальных координатах задается уравнением $x y+z^{2}+w^{3}=0$.

В обозначениях статьи $[1, \S 2]$, где описаны модели расслоений на поверхности дель Пещцо степени 1 при помоши двойных накрытий, расслоение $V_{C} \rightarrow \mathbb{P}^{1}$ имеет одни и те же структурные константы $\left(\varepsilon, n_{1}, n_{2}, n_{3}\right)=(0,0,1,2)$ для всех кривых $C$, в том числе и особых. Отметим, что особые точки на $V_{C}$ возникают из соответствующих двойных точек на дивизоре ветвления (если использовать упомянутую модель в виде двойного накрытия). Кроме того, неособые расслоения на поверхности дель Пещц степени 1 обладают свойством единственности неособой модели в классе бирациональных отображений над базой (см. [2, следствие 4.5]). Все перечисленное выше может быть сформулировано в виде следуюшей гипотезы.

ГИПотеЗА 1.1. Пусть $V$ - неособое трехмерное многообразие Фано, являющееся двойным накрытием конуса над поверхностью Веронезе. Тогда множсество

$$
\left\{V ; V_{C} \rightarrow \mathbb{P}^{1}, C \in \mathscr{L}\right\}
$$

исчерпьвает все структуры Мори для $V$.

Первые шаги в направлении доказательства этой гипотезы ${ }^{1}$ были сделаны в работах [3] и [6]. В [3] было показано, что кривые, являющиеся центрами максимальных особенностей линейных систем на $V$, исчерпываются семейством $\mathscr{L}$, что как раз соответствует переходу $V_{C} \rightarrow V$ на другую структуру Мори. Автор статьи [6] попытался доказать, что линейные системы не могут иметь максимальных особенностей над точками (и этого, с учетом результатов [1], почти достаточно для доказательства гипотезы), но предложенное им доказательство содержит серьезную

\footnotetext{
${ }^{1}$ Примечание при корректуре. К моменту выхода настоящей статьи гипотеза 1.1 автором доказана и будет опубликована в ближайшее время.
} 
ошибку. Точнее, исключая в $[6, \S 3]$ бесконечно близкую максимальную особенность над особыми точками кривых из $\mathscr{L}$, он строит пробный класс, не являющийся таковым на самом деле. Если $B_{0}$ - особая точка кривой $C \in \mathscr{L}$, то единственный элемент из $|H-C|$, имеющий особую точку в $B_{0}$, обязательно будет базисной компонентой для предлагаемого в [6] пробного класса (чего быть ни в коем случае не должно).

В настояшей статье предпринята попытка разобраться в этом вопросе. Выясняется, что линейные системы на $V$ если и имеют максимальные особенности над точками, то очень простого и конкретного вида (см. п. 2) теоремы 3.1). При этом вопрос о существовании таких особенностей пока остается открытым.

Статья организована следующим образом. В $\S 2$ напоминаются ставшие недавно известными результаты о структуре максимальных особенностей. В $\S 3-5$ приведено доказательство основного результата (теорема 3.1) работы. Статью завершает $\S 6$, где кратко обсуждаются вопросы, решение которых необходимо для доказательства гипотезы 1.1.

\section{§2. О максимальных особенностях линейных систем}

Предположим, что $X$ является трехмерным проективным многообразием с $\mathbb{Q}$-фракториальными терминальными особенностями, $\mathscr{D}$ - линейная система без неподвижных компонент, имеющая максимальную особенность. Другими словами, существует нормирование $\nu$ поля функций на $X$ такое, что для него выполнено неравенство Нётера-Фано

$$
\nu(\mathscr{D})>\mu \delta_{\nu}
$$

где $\delta_{\nu}$ - каноническая кратность относительно $\nu$, а $\mu$ - порог канонического присоединения. Это же можно выразить иначе: пара $K_{X}+\frac{1}{\mu} \mathscr{D}$ имеет неканонические особенности. Можно предполагать, что $\mathscr{D}$ является собственным прообразом некоторой (например, очень обильной) линейной системы при бирациональном отображении $X$ на какое-то многообразие.

С точки зрения программы минимальных моделей, такая ситуация приводит к следуюшему $[7, \S 2]$. Пусть $c$ - минимальное положительное число такое, что пара $K_{X}+\frac{1}{c} \mathscr{D}$ канонична, $e(X, \mathscr{D})$ - число всех различных нормирований поля функций $X$, относительно которых кратность $K_{X}+\frac{1}{c} \mathscr{D}$ равна нулю. Заметим, что $c>\mu$ по условию. Тогда существует максимальное крепантное разрешение $\varphi: Y \rightarrow X$ такое, что $K_{Y}+\frac{1}{c} \mathscr{D}_{Y}=\varphi^{*}\left(K_{X}+\frac{1}{c} \mathscr{D}\right)$ и $\rho(Y / X)=e(X, \mathscr{D})$. Другими словами, $\varphi$ "вытягивает" только дивизоры с нулевыми дискрепантностями для пары $K_{X}+\frac{1}{c} \mathscr{D}$. Далее, относительная программа минимальных моделей для $Y / X$ (относительно $\left.K_{Y}\right)$ должна оборваться через конечное число шагов, и последним шагом будет экстремальное дивизориальное стягивание $\psi: Z \rightarrow X$. Заметим, что морфизм $\psi$ является крепантным для $K_{Z}+\frac{1}{c} \mathscr{D}_{Z}$, т.е. $K_{Z}+\frac{1}{c} \mathscr{D}_{Z}=\psi^{*}\left(K_{X}+\frac{1}{c} \mathscr{D}\right)$. Поскольку $c>\mu$, то исключительный дивизор $E$ стягивания $\psi$ реализует нормирование поля функций $X$, относительно которого пара $K_{X}+\frac{1}{\mu} \mathscr{D}$ неканонична, т. е. имеет максимальную особенность. Таким образом, в методе максимальных особенностей линейных систем можно использовать следующий принцип.

Пусть линейная система $\mathscr{D}$ имеет максимальную особенность относительно некоторого нормирования $\nu$ поля функций на $X$. Тогда существует нормирование $\nu^{\prime}$, 
реализуемое экстремальным раздутием в категории Мори, относительно которого $\mathscr{D}$ также имеет максимальную особенность. Другими словами, можно сразу предполагать, что максимальная особенность линейной системы есть экстремальное дивизориальное раздутие, относительно которого пара $K_{X}+\frac{1}{\mu} \mathscr{D}$ неканонична.

Недавно был получен важный результат о структуре дивизориальных стягиваний в неособые точки [8].

ПРЕДЛОЖЕНИЕ 2.1. Пусть $\psi: Z \rightarrow X$ - экстремальное стягивание (в категории Мори) дивизора $E$ в неособую точку $P \in X$. Тогда для подходящих локальных координат $(x, y, z)$ на $X$ морфизм $\psi$ является взвешенным раздутием с весами $(1, L, N), L \leqslant N$, числа $L$ и $N$ взаимно простьи.

В виде гипотезы этот результат был предположен в $[7$, п. 4.7]. Его важность заключается в следующем. Если линейная система $\mathscr{D}$ без неподвижных компонент имеет максимальную особенность указанного вида, то неравенство Нётера-Фано для нее выглядит следующим образом:

$$
\nu_{E}(\mathscr{D})>\mu(N+L)
$$

Тогда для общих элементов $D_{1}$ и $D_{2}$ линейной системы имеем

$$
\operatorname{mult}_{P} D_{1} \circ D_{2} \geqslant \nu_{E}(\mathscr{D})^{2} E^{2}>\mu^{2} \frac{(N+L)^{2}}{N L} \geqslant 4 \mu^{2} \text {. }
$$

Отсюда, в частности, немедленно следует результат Исковских и Манина о нерациональности гладкой трехмерной квартики.

Другое важное следствие предложения 2.1 касается структуры графа Г максимальной особенности. Напомним, что любое нормирование $\nu$ поля функций может быть реализовано цепочкой раздутий

$$
X_{N} \stackrel{\varphi_{N}}{\longrightarrow} X_{N-1} \stackrel{\varphi_{N-1}}{\longrightarrow} \cdots \stackrel{\varphi_{2}}{\longrightarrow} X_{1} \stackrel{\varphi_{1}}{\longrightarrow} X_{0}=X
$$

с исключительными дивизорами $E_{i} \subset X_{i}$ и центрами раздутий $B_{i} \subset E_{i}$. Таким образом, можно полагать, что $B_{0}, B_{1}, \ldots, B_{L-1}$ - точки, $B_{L}, \ldots, B_{N-1}$ - кривые. Дивизор $E_{N}$ реализует нормирование $\nu$. Граф Г является ориентированным графом, состояшим из $N$ вершин. По определению в $\Gamma$ существует стрелка $j \rightarrow i$, если $j>i$ и $B_{j-1} \subset E_{i}^{j-1}$ (верхние индексы обозначают собственные прообразы на соответствующих этажах цепочки раздутий). Заметим, что всегда существуют стрелки $i+1 \rightarrow i$.

Предположим теперь, что граф $\Gamma$ соответствует взвешенному раздутию точки $B_{0}$ при подходящем выборе локальных координат $(x, y, z)$ на $X, B_{0}=\{x=$ $y=z=0\}$. Тогда нетрудно видеть, что $\Gamma$ является цепочкой, т. е. сушествуют только стрелки $i+1 \rightarrow i$, а взвешенное раздутие имеет веса $(1, L, N)$. Кроме того, кривые $B_{L+1}, \ldots, B_{N-1}$ есть сечения соответствующих линейчатых поверхностей $E_{L+1}, \ldots, E_{N-1}$, а $B_{L} \subset E_{L} \cong \mathbb{P}^{2}$ является прямой.

В локальных координатах уравнением $z=0$ определяется росток неособого в точке $B_{0}$ дивизора, собственные прообразы которого содержат все центры раздутий $B_{i}$. Уравнением $y=0$ на этом дивизоре высекается росток неособой кривой, проходящей через все (бесконечно близкие) точки $B_{1}, \ldots, B_{L-1}$. 
Тем же способом, что и в [10], можно показать (с учетом свойств графа $Г$ ), что для общих элементов $D_{1}$ и $D_{2}$ линейной системы $\mathscr{D}$ без неподвижных компонент, имеющей максимальную особенность с графом $Г$, выполнено

$$
\sum_{i=0}^{L-1} \operatorname{mult}_{B_{i}}\left(D_{1} \circ D_{2}\right)^{i} \geqslant \sum_{i=1}^{N} \nu_{i}^{2}
$$

где $\nu_{i}=\operatorname{mult}_{B_{i-1}} \mathscr{D}^{i-1}$. Неравенство Нётера-Фано в этом случае выглядит следующим образом:

$$
\sum_{i=1}^{N} \nu_{i}>\mu(L+N)
$$

Отсюда легко вывести квадратичное неравенство метода максимальных особенностей:

$$
\sum_{i=0}^{L-1} \operatorname{mult}_{B_{i}}\left(D_{1} \circ D_{2}\right)^{i}>4 \mu^{2} L+\mu^{2} \frac{(N-L)^{2}}{N} .
$$

Наконец, заметим, что конфигурация центров раздутий и исключительных дивизоров обладает еще одним важным свойством, а именно можно полагать, что кривая $B_{L+1}$ (если она существует) не пересекает собственный прообраз $E_{L}$, т. е. $B_{L+1} \cap E_{L}^{L+1}=\varnothing$.

Действительно, в противном случае легко видеть, что $\nu_{L-1} \geqslant \nu_{L}+\nu_{L+1}>2 \mu$, откуда $\nu_{1}>2 \mu$, т. е. мы уже имеем простейшее нормирование, реализуемое обыкновенным раздутием точки $B_{0}$, относительно которого $\mathscr{D}$ имеет максимальную особенность.

\section{§ 3. Формулировка основного результата и начало его доказательства}

Пусть $V$ - двойной конус над поверхностью Веронезе, $\operatorname{Pic}(V)=\mathbb{Z} H, K_{V} \sim$ $-2 H$, группа $A^{2}(V)$ одномерных циклов порождена классом $l$, т.е. $A^{2}(V)=\mathbb{Z} l$, $\mathscr{L}$ - двумерное семейство кривых класса $l$.

Предположим, что существует бирациональное отображение $\chi: V \rightarrow U$ на расслоение Мори $\rho: U \rightarrow S$. Пусть $\mathscr{D}_{U}=\left|r^{\prime}\left(-K_{U}\right)+\rho^{*}(A)\right|-$ очень обильная линейная система на $U, A$ - обильный дивизор на $S$ и $\mathscr{D}=\chi_{*}^{-1} \mathscr{D}_{U}-$ собственньй прообраз $\mathscr{D}_{U}$ на $V$. Можно полагать, что $\mathscr{D} \subset\left|r\left(-K_{V}\right)\right|$. Тогда, как это следует из [7, теорема 4.2], $r \geqslant r^{\prime}$, и если $\chi$ не изоморфизм, то $r>r^{\prime}$. Следовательно, пара $K_{V}+\frac{1}{r} \mathscr{D}$ неканонична, т. е. $\mathscr{D}$ имеет максимальную особенность относительно некоторого нормирования $\nu$ поля функций на $V$. Положим $n=2 r$, так что $\mathscr{D} \subset$ $|n H|$.

Пусть $B_{0}$ является центром $\nu$ на $V$. Можно полагать, что $\nu$ реализуется экстремальным дивизориальным раздутием $\psi: Z \rightarrow X$. Обозначим Г граф максимальной особенности, $B_{i}$ - центры соответствующих раздутий. Если $B_{0}-$ кривая, то $\psi$ не что иное, как обыкновенное раздутие кривой (по крайней мере, в неособой части $\left.B_{0}\right)$. Если же $B_{0}$ - точка, то полагаем $\psi$ взвешенным раздутием с весами $(1, L, N)$, так что Г имеет $N$ вершин, из которых первые $L$ соответствуют раздутиям точек $B_{0}, \ldots, B_{L-1}$, а последние $N-L$ - раздутиям кривых $B_{L}, \ldots, B_{N-1}$. Напомним, что Г не имеет инцидентностей, т. е. существуют только стрелки $i+1 \rightarrow i$. 
Теорема 3.1. 1) Предположим, что $B_{0}$ - кривая. Тогда $B_{0} \in \mathscr{L}, \psi$ - раздутие пучка идеалов $B_{0}$ (напомним, что $B_{0}$ является полным пересечением в этом случае), $Z$ - одно из расслоений Мори на поверхности дель Пеццо степени 1, описанных в гипотезе 1.1.

2) Предположим, что $B_{0}$ - точка и $\mathscr{D}$ не имеет максимальных особенностей вдоль кривых. Тогда $B_{0} \neq P=\operatorname{Bas}|H|$, поэтому существует единственная кривая $l_{0} \in \mathscr{L}$, содержащая $B_{0}$. Далее, $l_{0}$ имеет особенность в $B_{0}$, причем:

а) если $B_{0}-$ нодальная точка $l_{0}$, то $L=1 u 2 \leqslant N \leqslant 5$;

б) если $B_{0}-$ каспидальная точка $l_{0}$, то $L=1$ и $N=2$.

ЗАмЕчАниЕ 3.2. Возможно, п. 2) теоремы 3.1 лишний, т. е. максимальных особенностей указанного вида не существует.

ДокАзАТЕЛЬСтво. Случай 1$)$, т. е. когда $B_{0}$ - кривая, был разобран в работе [3]. В дальнейшем будем предполагать, что $B_{0}$ - точка.

Покажем сначала, что $B_{0} \neq P$. В рассматриваемом случае порог канонического присоединения есть $r=\frac{1}{2} n$, так что согласно формулам из $\S 2$ для общих элементов $D_{1}$ и $D_{2}$ линейной системы $\mathscr{D}$ вьполнено

$$
\operatorname{mult}_{B_{0}} D_{1} \circ D_{2}>\left(\frac{n}{2}\right)^{2} \frac{(L+N)^{2}}{L N}>n^{2}
$$

откуда если $B_{0}=P$, то из пересечения $D_{1}$ и $D_{2}$ с общим элементом $S \in|H|$ (напомним, что $P=\operatorname{Bas}|H|$ ) получаем

$$
n^{2}=D_{1} \circ D_{2} \circ S \geqslant \text { mult }_{B_{0}} D_{1} \circ D_{2}>n^{2} .
$$

Противоречие.

Итак, $B_{0} \neq P$, и существует единственная кривая $l_{0} \in \mathscr{L}$, содержащая точку $B_{0}$. Положим

$$
D_{1} \circ D_{2}=\alpha l_{0}+Z_{0},
$$

где $Z_{0}$ - эффективньй 1 -цикл, $l_{0} \not \subset \operatorname{Supp} Z_{0}$. Поскольку $D_{1} \circ D_{2} \sim n^{2} l$, то, очевидно, $Z_{0} \sim\left(n^{2}-\alpha\right) l_{0}$. Заметим, что для обшего элемента $S \in\left|H-B_{0}\right|$ имеем

$$
\text { mult }_{B_{0}} Z_{0} \leqslant Z_{0} \circ S=n^{2}-\alpha .
$$

Предположим, что $B_{0}$ - неособая точка $l_{0}$. Тогда

$$
\operatorname{mult}_{B_{0}} D_{1} \circ D_{2} \leqslant \alpha+n^{2}-\alpha=n^{2} .
$$

С другой стороны, из квадратичного неравенства (2.2) следует,что

$$
\operatorname{mult}_{B_{0}} D_{1} \circ D_{2}>4\left(\frac{n}{2}\right)^{2}=n^{2}
$$

Таким образом, $B_{0}$ должна быть особой точкой $l_{0}$. Предположим вначале, что взвешенное раздутие $\psi$ имеет веса $(1,1,1)$, т.е. $L=N=1$. Нетрудно видеть, что $\left|H-B_{0}\right|$ содержит единственный элемент, скажем $S$, имеющий особую точку 
в $B_{0}$. Общий дивизор $G \sim 2 H$, проходящий через точку $B_{0}$, высекает на $S$ неприводимую кривую $C$ класса $2 l$, причем $C$ имеет двойную точку в $B_{0}$. Неравенство Нётера-Фано для указанного раздутия имеет вид $\nu_{1}>n$, и мы для общего $D \in \mathscr{D}$ получаем

$$
2 n=C \circ D \geqslant \text { mult }_{B_{0}} C \cdot \text { mult }_{B_{0}} D=2 \nu_{1}>2 n .
$$

\section{Противоречие.}

Итак, показано, что $B_{0}$ является двойной точкой $l_{0}$ (нодальной либо каспидальной) и взвешенное раздутие $\psi$ не может иметь веса $(1,1,1)$. Таким образом, в дальнейшем полагаем $1 \leqslant L<N$ (напомним, что $L$ и $N$ должны быть взаимно просты).

Обозначим при $1 \leqslant i \leqslant L$

$$
c_{i}=\operatorname{mult}_{B_{i-1}} Z_{0}^{i-1}
$$

где $Z_{0}^{j}$ - собственные прообразы $Z_{0}$ на соответствующих этажах цепочки раздутий. Очевидно, $c_{1} \geqslant c_{2} \geqslant \cdots \geqslant c_{L}$. Далее, положим

$$
k=\max _{1 \leqslant i \leqslant L}\left\{i: B_{i-1} \in l_{0}^{i-1}\right\} .
$$

Заметим, что для общего элемента $S \in\left|H-B_{0}\right|=\left|H-l_{0}\right|$ имеем $B_{k-1} \in S^{k-1}$, так что

$$
c_{1}+\cdots+c_{k} \leqslant Z_{0} \circ S=n^{2}-\alpha .
$$

В любом случае выполняется $c_{i} \leqslant n^{2}-\alpha$.

Поскольку $B_{0}$ является двойной точкой $l_{0}$, неравенство $(2.2)$ перепишется в виде

$$
(k+1) \alpha+c_{1}+\cdots+c_{L}>n^{2} L+\frac{n^{2}}{4} \frac{(N-L)^{2}}{N} .
$$

Предположим, что $k<L$. Поскольку $c_{i} \leqslant n^{2}-\alpha$, то из неравенства (3.2) следует

$$
L n^{2} \geqslant(k+1) \alpha+L\left(n^{2}-\alpha\right)>L n^{2} .
$$

Противоречие.

В дальнейшем будем всегда полагать $k=L$. В силу неравенств (3.1) и (3.2) имеем

$$
(L+1) \alpha+n^{2}-\alpha>n^{2} L+\frac{n^{2}}{4} \frac{(N-L)^{2}}{N},
$$

откуда получаем необходимое для дальнейших рассуждений основное неравенство

$$
\alpha>\frac{(L-1)}{L} n^{2}+n^{2} \frac{(N-L)^{2}}{4 N L} .
$$




\section{§4. Продолжение доказательства основной теоремы. Случай $l_{0}$ с нодальной особой точкой, $k=L<N$}

В этом параграфе предполагаем, что $B_{0} \in l_{0}$ является "нодой", т. е. обыкновенной двойной точкой.

Пусть $k=L=1$. Тогда, как это видно из (3.3), при $N \geqslant 6$ достигается противоречие с условием $\alpha \leqslant n^{2}$. Чтобы завершить доказательство п. 2), а) теоремы 3.1 , остается показать, что случаи $k=L>1$ не реализуются. Будем использовать оценки сверху усредненных кратностей

$$
\theta_{i}=\frac{1}{i}\left(\nu_{1}+\cdots+\nu_{i}\right)
$$

Заметим, что $\nu_{1}=\theta_{1} \geqslant \theta_{2} \geqslant \theta_{3} \geqslant \cdots$. Необходимые нам оценки получаются из следующих соображений.

Выберем обшие элементы $S \in\left|H-l_{0}\right|$ и $D \in \mathscr{D}$. Заметим, что

$$
\mu \stackrel{\text { def }}{=} \operatorname{mult}_{l_{0}} D=\left.\operatorname{ord}_{l_{0}} D\right|_{S} \text {. }
$$

По условию линейная система $\mathscr{D}$ не имеет максимальных особенностей вдоль кривых, поэтому далее будем предполагать, что $\mu \leqslant \frac{n}{2}$. Положим

$$
\left.D\right|_{S}=\mu l_{0}+C
$$

где $l_{0} \not \subset \operatorname{Supp} C$. Далее, на многообразии $V_{1}$ имеем

$$
\left.D^{1}\right|_{S^{1}}=\mu l_{0}^{1}+C^{1}+m_{1} e_{1}
$$

где $e_{1}=E_{1} \cap S^{1}$ и $m_{1} \geqslant 0$. Продолжая таким образом, на многообразии $V_{i}$, $i \leqslant L-1$, будем иметь

$$
\left.D^{i}\right|_{S^{i}}=\mu l_{0}^{i}+C^{i}+m_{1} e_{1}^{i}+\cdots+m_{i-1} e_{i-1}^{i}+m_{i} e_{i}
$$

где $e_{j}=E_{j} \cap S^{j}$ и $m_{j}=\left.\operatorname{ord}_{e_{j}} D^{j}\right|_{S^{j}}$. Поскольку граф Г не имеет инщидентностей, конфигурация исключительных кривых $\left\{e_{1}^{i}, e_{2}^{i}, \ldots, e_{i}\right\}$ на $S^{i}$ имеет вид цепочки, так что $B_{i} \in e_{i}$ и $B_{i} \notin e_{1}^{i} \cup e_{2}^{i} \cup \cdots \cup e_{i-1}^{i}$.

Обозначим $\alpha_{i}=\operatorname{mult}_{B_{i}} C^{i}$ и $\widetilde{\nu}_{i}=\left.\operatorname{mult}_{B_{i-1}} D^{i-1}\right|_{S^{i-1}}$. Легко видеть, что $\widetilde{\nu}_{i}=$ $\nu_{i}+m_{i}$. Кроме того,

$$
\begin{gathered}
\widetilde{\nu}_{1}=2 \mu+\alpha_{0}, \\
\widetilde{\nu}_{2}=\mu+\alpha_{1}+m_{1}, \\
\ldots \ldots \ldots \ldots \ldots \ldots \ldots \ldots \ldots \ldots \\
\widetilde{\nu}_{L}=\mu+\alpha_{L-1}+m_{L-1} .
\end{gathered}
$$

Графически многообразия $V_{i}, i=0, \ldots, L-1$, представлены на рис. 1 . Отметим, что $m_{i}=\left.\operatorname{ord}_{e_{i}} D^{i}\right|_{S^{i}}=\operatorname{mult}_{e_{i}} D^{i}$ при $i \leqslant k$ ввиду выбора общей поверхности $S \in\left|H-l_{0}\right|$.

Суммируя приведенные выше равенства, находим

$$
\nu_{1}+\cdots+\nu_{L} \leqslant \nu_{1}+\cdots+\nu_{L}+m_{L}=(L+1) \mu+\alpha_{0}+\cdots+\alpha_{L-1} .
$$




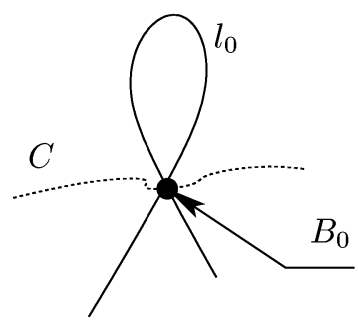

$V=V_{0}$

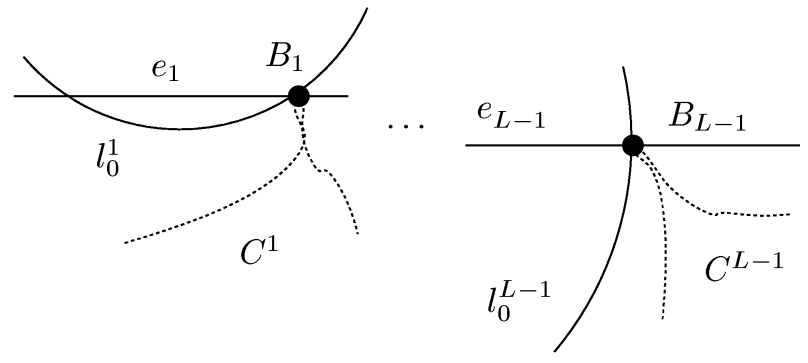

$V_{1}$

$V_{L-1}$

Рис. 1

Кроме того, поскольку $\alpha_{0} \geqslant \alpha_{1} \geqslant \cdots$ и $n-\mu=C \circ l_{0}=2 \alpha_{0}+\alpha_{1}+\cdots+\alpha_{L-1}$, получаем

$$
\alpha_{0}+\alpha_{1}+\cdots+\alpha_{L-1} \leqslant \frac{L}{L+1}(n-\mu) \leqslant(n-\mu) .
$$

Рассмотрим многообразие $V_{L}$ (см. рис. $\left.2, a\right)$. Дальнейшие рассуждения зависят от того, как расположена прямая $B_{L} \subset E_{L} \cong \mathbb{P}^{2}$.
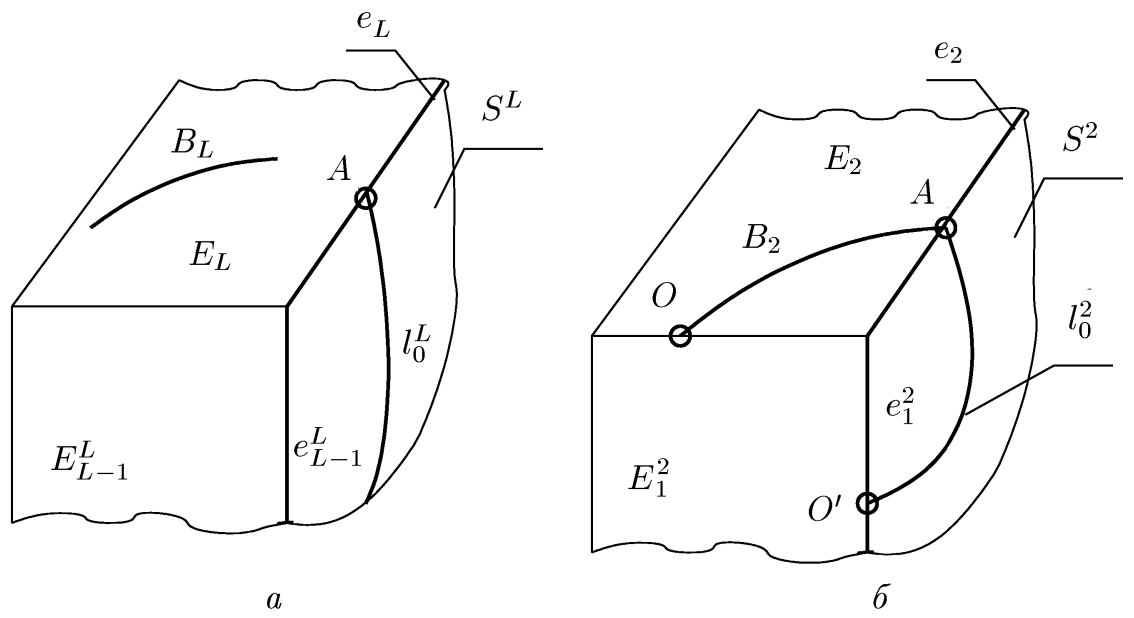

Рис. 2

Предположим сначала, что точка $A=l_{0}^{L} \cap e_{L}$ лежит вне $B_{L}$. Тогда из пересечения общей прямой, лежащей на $E_{L}$ и проходящей через точку $A$, и дивизора $D$ получаем, что $\nu_{L} \geqslant \nu_{L+1}+\mu$. Из оценок (4.1) и (4.2) видно, что

$$
L \theta_{L}=\nu_{1}+\cdots+\nu_{L} \leqslant(L+1) \mu+n-\mu=L \mu+n,
$$

т. е. $\theta_{L} \leqslant \mu+\frac{1}{2} n$. Однако $\nu_{L} \leqslant \theta_{L}$, так что

$$
\frac{n}{2}<\nu_{L+1} \leqslant \nu_{L}-\mu \leqslant \theta_{L}-\mu \leqslant \frac{n}{L}
$$

что противоречит условию $L>1$. 
Пусть теперь $B_{L}=e_{L}$, т.е. $B_{L}=S^{L} \cap E_{L}$. Тогда $m_{L}=\nu_{L+1}$, откуда $\nu_{L+1}+$ $\nu_{L}=\nu_{L}+m_{L}=\mu+\alpha_{L-1}+m_{L-1}$. Из неравенств (4.1) и (4.2) получаем

$$
\nu_{1}+\cdots+\nu_{L}+\nu_{L+1} \leqslant(L+1) \mu+\frac{L}{L+1}(n-\mu),
$$

так что

$$
\theta_{L+1} \leqslant \mu+\frac{L}{(L+1)^{2}}(n-\mu)
$$

и при $L \geqslant 2$ имеем

$$
\theta_{L+1} \leqslant \frac{2}{9} n+\frac{7}{9} \mu \leqslant \frac{2}{9} n+\frac{7}{18} n=\frac{11}{18} n .
$$

Эта оценка противоречит требованию $\theta_{i}>\frac{5}{8} n$ для всех $i$. Действительно, если $\theta_{i} \leqslant \frac{5}{8} n$, неравенство Нётера-Фано (2.1) влечет

$$
\frac{5}{8} n N>\frac{n}{2}(L+N)
$$

т. е. $N>4 L$, и тогда в силу оценки (3.3) при $L \geqslant 2$ получаем

$$
\alpha>\frac{L-1}{L} n^{2}+\frac{9}{16} n^{2}>n^{2} .
$$

Противоречие.

Итак, нам осталось предположить, что $A \in B_{L} \neq e_{L}$. Геометрическая ситуация в этом случае (при $L=2$ ) изображена на рис. 2,6 .

Отметим сначала, что $N>L+1$. Действительно, если $N=L+1$, то из неравенства Нётера-Фано находим, что при $L \geqslant 2$

$$
\theta_{L+1}>\frac{n}{2} \cdot \frac{2 L+1}{L+1} \geqslant \frac{5}{6} n
$$

С другой стороны, из оценок (4.1) и (4.2) следует, что

$$
\theta_{L+1} \leqslant \mu+\frac{n}{L+1} \leqslant \frac{n}{2}+\frac{n}{3}=\frac{5}{6} n .
$$

Итак, $N>L+1$, т. е. сушествует кривая $B_{L+1}$. По замечанию, приведенному в конце $\S 2, B_{L+1} \cap E_{L}^{L+1}=\varnothing$, поэтому точка $\widetilde{O}=B_{L+1} \cap E_{L-1}^{L+1}$ не лежит на дивизоре $E_{L}^{L+1}$. Тогда очевидно, что существует семейство неприводимых коник на $E_{L-1}$, проходяших через точки $B_{L-1}, O$ и $\widetilde{O}$, откуда вытекает важное для дальнейших рассуждений неравенство

$$
2 \nu_{L-1} \geqslant \nu_{L}+\nu_{L+1}+\nu_{L+2}
$$

Имеем два варианта взаимного расположения $B_{L+1}$ и $l_{0}^{L+1}$ :

1) либо $B_{L+1} \cap l_{0}^{L+1} \neq \varnothing$ (пересекаются в некоторой точке $\widetilde{B}_{L+1}$, лежащей на $\left.S^{L+1}\right)$;

2) либо $B_{L+1} \cap l_{0}^{L+1}=\varnothing$. 
Предположим сначала, что $B_{L+1} \cap l_{0}^{L+1} \neq \varnothing$. Тогда

$$
\widetilde{\nu}_{L+2}=\left.\operatorname{mult}_{\widetilde{B}_{L+1}} D^{L+1}\right|_{S^{L+1}}=\mu+\alpha_{L+1}+m_{L+1}
$$

где, как и выше, $m_{L+1}=\widetilde{\nu}_{L+1}-\nu_{L+1}$. Следовательно,

$$
\nu_{1}+\cdots+\nu_{L+2} \leqslant(L+3) \mu+\alpha_{0}+\cdots+\alpha_{L+1} \leqslant(L+3) \mu+\frac{L+2}{L+3}(n-\mu) .
$$

С другой стороны, пусть $2 \beta=\nu_{L+1}+\nu_{L+2}$. Тогда из неравенства (4.3) следует, что $2 \nu_{L-1} \geqslant 3 \beta$, и так как $\nu_{1}+\cdots+\nu_{L+2} \geqslant(L-1) \nu_{L-1}+\nu_{L}+\nu_{L+1}+\nu_{L+2}$, находим

$$
\frac{3}{2}(L+1) \beta \leqslant(L+3) \mu+\frac{L+2}{L+3}(n-\mu),
$$

откуда, учитьвая то, что $\mu \leqslant \frac{n}{2}$, получаем

$$
\beta \leqslant \frac{L+4}{3(L+1)} n
$$

Ранее мы использовали оценку

$$
\theta_{L} \leqslant \mu+\frac{n}{L} \leqslant\left(\frac{L+2}{2 L}\right) n .
$$

Из неравенства Нётера-Фано $\nu_{1}+\cdots+\nu_{N}>\frac{n}{2}(L+N)$ следует, что

$$
L \theta_{L}+(N-L) \beta>\frac{n}{2}(L+N)
$$

Подставляя сюда оценки для $\theta_{L}$ и $\beta$, находим

$$
\frac{5-L}{6(L+1)} N>\left(\frac{L+4}{3 L+3}\right) L-1
$$

что возможно только при $L=2$. Но в этом случае можно усилить наши оценки. В самом деле, из рис. 2,6 видно, что $l_{0}^{2}$ и $E_{1}^{2}$ пересекаются в точке $O^{\prime}$, поэтому можно выбрать семейство неприводимых коник на $E_{1}$, проходящих через точки $B_{1}, O, \widetilde{O}$ и $O^{\prime}$, откуда получаем

$$
2 \nu_{1} \geqslant \nu_{2}+\nu_{3}+\nu_{4}+\mu \text {. }
$$

Пусть $3 \beta=\nu_{2}+\nu_{3}+\nu_{4}$, и предположим, что $\beta>\frac{4}{7} n$. Тогда $2 n \geqslant 2 \nu_{1} \geqslant 3 \beta+$ $\mu>\frac{12}{7} n+\mu$, так что $\mu<\frac{2}{7} n$. В этом случае

$$
\nu_{1}+\nu_{2}+\nu_{3} \leqslant 4 \mu+\alpha_{0}+\alpha_{1}+\alpha_{2} \leqslant 4 \mu+\frac{3}{4}(n-\mu)<\frac{47}{28} n,
$$

т. е. $\theta_{3}<\frac{47}{84} n$, что противоречит ранее доказанному условию $\theta_{i}>\frac{5}{8} n$ для всех $i$. Таким образом, $\beta \leqslant \frac{4}{7} n$. Из неравенства Нётера-Фано $\nu_{1}+\cdots+\nu_{N}>\frac{n}{2}(N+2)$ и условия $\nu_{1} \leqslant n$ находим, что

$$
(N-1) \beta \geqslant \nu_{2}+\cdots+\nu_{N}>\frac{n}{2} N
$$


откуда $N>8$. Подставив в квадратичное неравенство (3.3) условия $L=2, N>8$, получаем

$$
\alpha>\frac{1}{2} n^{2}+\frac{(N-2)^{2}}{8 N} n^{2}>\frac{1}{2} n^{2}+\frac{36}{64} n^{2}>n^{2} .
$$

Противоречие.

Осталось рассмотреть случай $B_{L+1} \cap l_{0}^{L+1}=\varnothing$, который исключается точно такими же рассуждениями, с той лишь разнищей, что $\widetilde{\nu}_{L+2}=\alpha_{L+1}+m_{L+1}$, причем $\alpha_{L+1} \leqslant \frac{n-\mu}{L+1}$. Нетрудно заметить, что получаемые оценки при этом сильнее, чем в случае $B_{L+1} \cap l_{0}^{L+1} \neq \varnothing$.

Утверждение 2), а) теоремы 3.1 полностью доказано.

\section{§5. Завершение доказательства основной теоремы.}

Случай $l_{0}$ с каспидальной особой точкой, $k=L<N$

В этом параграфе предполагаем, что $l_{0}$ имеет каспидальную особую точку. Будем использовать обозначения, введенные в $\S 4$.

Выберем обший элемент $D \in \mathscr{D}$ и рассмотрим ограничения $\left.D^{i}\right|_{S^{i}}$, где $S^{i}$ - собственные прообразы обшего элемента $S \in\left|H-l_{0}\right|$. Графически многообразия $V_{0}$, $V_{1}$ и $V_{2}$ представлены на рис. 3 .

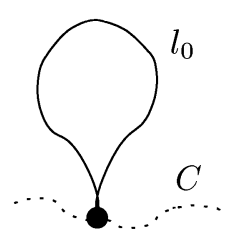

$B_{0}$

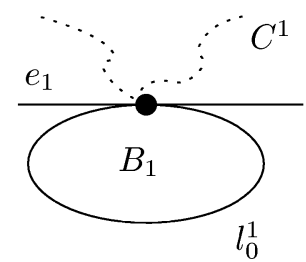

$V_{1}$

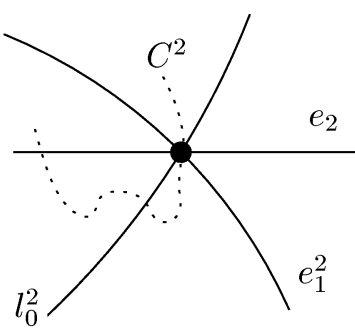

$V_{2}$

Рис. 3

Предположим сначала, что $L \geqslant 3$. Тогда, поскольку граф̆ $Г$ не имеет инцидентностей, $B_{2} \notin E_{1}^{2}$. Обозначая, как и ранее, $e_{i}=\left.E_{i}\right|_{S^{i}}$, имеем $B_{2} \notin e_{2}$, и тогда из рис. 3 видно, что

$$
\begin{aligned}
& \widetilde{\nu}_{1}=2 \mu+\alpha_{0}, \\
& \widetilde{\nu}_{2}=\mu+\alpha_{1}+m_{1}, \\
& \widetilde{\nu}_{3}=\alpha_{2}+m_{2} .
\end{aligned}
$$

Таким образом,

$$
\nu_{1}+\nu_{2}+\nu_{3} \leqslant 3 \mu+\alpha_{0}+\alpha_{1}+\alpha_{2} \leqslant 3 \mu+2 \alpha_{0}+\alpha_{1} \leqslant 2 \mu+n \leqslant 2 n,
$$

откуда $\theta_{2} \leqslant \frac{2}{3} n$. Тогда из неравенства Нётера-Фано имеем

$$
\frac{2}{3} N>\frac{n}{2}(L+N)
$$


так что $N>3 L$. Подставляя это в $(3.3)$, при $L \geqslant 3$ получаем

$$
\alpha>\left(\frac{L-1}{L}\right) n^{2}+\frac{1}{3} n^{2} \geqslant n^{2} .
$$

Противоречие.

Итак, полагаем $L \leqslant 2$. Пусть сначала $L=2$ (см. рис. 4 ).

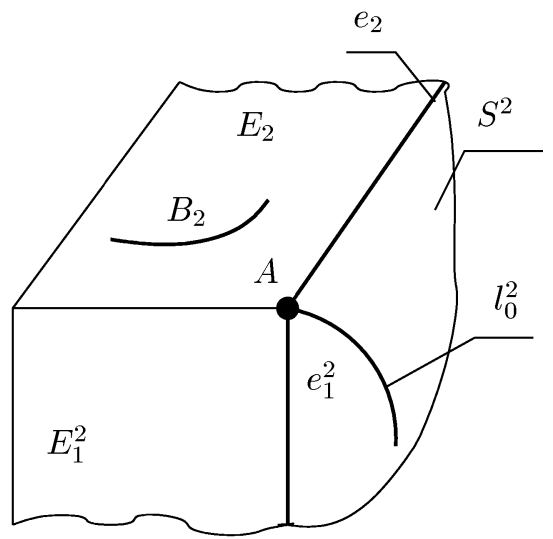

Рис. 4

Возможны три случая: $A \notin B_{2}, A \in B_{2}=e_{2}$ и $A \in B_{2} \neq e_{2}$.

Пусть $A \notin B_{2}$. Тогда из пересечения $D^{2}$ с общей прямой на $E_{2}$, проходящей через точку $A$, находим

$$
\nu_{2} \geqslant \nu_{3}+\mu .
$$

С другой стороны, $\nu_{1}+\nu_{2} \leqslant 3 \mu+\alpha_{0}+\alpha_{1} \leqslant 3 \mu+n-\mu=n+2 \mu$, откуда $\theta_{2} \leqslant \mu+\frac{n}{2}$. Тогда

$$
\frac{n}{2}<\nu_{3} \leqslant \nu_{2}-\mu \leqslant \theta_{2}-\mu \leqslant \frac{n}{2} .
$$

Пусть теперь $B_{2}=e_{2}=S^{2} \cap E_{2}$. Заметим, что при общем выборе поверхности $S$

$$
m_{2}=\left.\operatorname{ord}_{e_{2}} D^{2}\right|_{S^{2}}=\operatorname{mult}_{e_{2}} D^{2}=\nu_{3} .
$$

Однако

$$
\nu_{1}+\nu_{2}+m_{2}=3 \mu+\alpha_{0}+\alpha_{1},
$$

так что

$$
\nu_{1}+\nu_{2}+\nu_{3} \leqslant 3 \mu+\frac{2}{3}(n-\mu) \leqslant \frac{11}{6} n,
$$

и мы получаем $\theta_{3} \leqslant \frac{11}{18} n$. Подставляя это в неравенство Нётера-Фано, находим

$$
\frac{11}{18} n N>\frac{n}{2}(N+2)
$$


т. е. $N>9$, и из неравенства (3.3) при $L=2, N \geqslant 9$ получаем

$$
\alpha>\frac{1}{2} n^{2}+\frac{49}{72} n^{2}>n^{2} .
$$

Противоречие.

Пусть, наконец, $A \in B_{2} \neq e_{2}$. Случай $N \geqslant 9$ исключается неравенством (3.3), так что ввиду взаимной простоты $L=2$ и $N$ рассмотрим лишь случаи $N \in$ $\{3,5,7\}$. Предположим, что $N=3$. Пусть $A^{\prime}$ - точка пересечения $l_{0}^{3}$ и $E_{3}$. Тогда нетрудно убедиться в том, что на $E_{1}$ существует семейство неприводимых коник, проходящих через точки $B_{1}, A$ и $A^{\prime}$, и из пересечения такой коники с $D^{3}$ находим

$$
2 \nu_{1} \geqslant \nu_{2}+\nu_{3}+\mu
$$

$\mathrm{C}$ другой стороны, $\widetilde{\nu}_{3}=\mu+m_{1}+m_{2}$, так что

$$
2 \nu_{1}+\nu_{2}+\nu_{3} \leqslant 6 \mu+2 \alpha_{0}+\alpha_{1}+\alpha_{2} \leqslant 6 \mu+n-\mu=n+5 \mu,
$$

откуда $2\left(\nu_{2}+\nu_{3}\right) \leqslant n+4 \mu \leqslant 3 n$, т. е. $\nu_{2}+\nu_{3} \leqslant \frac{3}{2} n$. Однако из неравенства Нётера-Фано имеем оценку

$$
\nu_{1}+\nu_{2}+\nu_{3}>\frac{5}{2} n
$$

откуда получаем противоречие: $\nu_{1}>n$. Пусть теперь $N=5$ или $N=7$. Нас интересуют следующие случаи взаимного расположения кривых $B_{3}, e_{1}^{3}, l_{0}^{3}$ :

1) $B_{3} \cap l_{0}^{3} \neq \varnothing$;

2) $B_{3} \cap e_{1}^{3} \neq \varnothing$;

3) $B_{3} \cap l_{0}^{3}=\varnothing, B_{3} \cap e_{1}^{3}=\varnothing$.

Напомним, что кривая $B_{2}$ проходит через точку $A$ (рис. 4 ).

Сначала предположим, что сушествует точка $A^{\prime}=B_{3} \cap l_{0}^{3}$. Тогда, очевидно, на многообразии $V_{4}$ сушествует точка пересечения $A^{\prime \prime}=E_{4} \cap l_{0}^{4}$. Набор $\left(B_{1}, A, A^{\prime}, A^{\prime \prime}\right)$ можно рассматривать как конфигурацию из четырех (бесконечно близких) точек на $E_{1} \cong \mathbb{P}^{2}$ и нетрудно убедиться в том, что эта конфигурация определяет одномерное семейство коник на $E_{1}$ с неприводимым обшим элементом $T$, откуда получаем неравенство

$$
2 \nu_{1}=D^{1} \circ T \geqslant \nu_{2}+\nu_{3}+\nu_{4}+\mu .
$$

Учитывая, что $\widetilde{\nu}_{4}=\mu+\alpha_{3}+m_{3}$, находим

$$
2 \nu_{1}+\nu_{2}+\nu_{3}+\nu_{4} \leqslant 7 \mu+2 \alpha_{0}+\alpha_{1}+\alpha_{2}+\alpha_{3} \leqslant 7 \mu+n-\mu=n+6 \mu,
$$

а так как $2 \nu_{1} \geqslant \nu_{2}+\nu_{3}+\nu_{4}+\mu$, то

$$
2\left(\nu_{2}+\nu_{3}+\nu_{4}\right) \leqslant n+5 \mu \leqslant \frac{7}{2} n .
$$

Положим $\beta_{i}=\frac{1}{i}\left(\nu_{2}+\nu_{3}+\cdots+\nu_{i+1}\right)$. Заметим, что $\beta_{2} \geqslant \beta_{3} \geqslant \beta_{4} \geqslant \cdots$.

Итак, $\beta_{3} \leqslant \frac{7}{12} n$. С другой стороны, из неравенства Нётера-Фано следует, что $\nu_{1}+4 \beta_{4}>\frac{7}{2} n$ при $N=5$ и $\nu_{1}+6 \beta>\frac{9}{2} n$ при $N=7$. Однако $\nu_{1} \leqslant n$, откуда $\beta_{4}>\frac{5}{8} n$ 
при $N=5$ и $\beta_{6}>\frac{7}{12} n$ при $N=7$. Обе оценки снизу противоречат полученному условию $\beta_{3} \leqslant \frac{7}{12} n$.

Теперь предположим, что существует точка пересечения $A^{\prime \prime}=B_{3} \cap e_{1}^{3}$, и обозначим $A^{\prime}=l_{0}^{3} \cap E_{3}$. Поскольку $e_{1}^{3} \cap l_{0}^{3}=\varnothing$, а точки $A^{\prime}$ и $A^{\prime \prime}$ лежат в одном слое линейчатой поверхности $E_{3}$, то $A^{\prime}$ не лежит на кривой $B_{3}$, являющейся сечением $E_{3}$. Далее, обозначим $s$ и $f$ классы исключительного сечения и слоя поверхности $E_{3}$, имеющей тип $\mathbb{F}_{2}$. Нетрудно вычислить, что

$$
\left.D^{3}\right|_{E_{3}} \sim \nu_{3} s+\left(\nu_{2}+\nu_{3}\right) f
$$

Заметим, что эффективньй цикл $\left.D_{3}\right|_{E_{3}}$ содержит кривую $B_{3} \sim s+2 f$ с кратностью не ниже $\nu_{4}$. Выберем общую кривую $T \sim s+2 f$, проходящую через точку $A^{\prime}$. Поскольку $A^{\prime} \notin B_{3}$, кривая $T$ пересечет $B_{3}$ в двух отличных от $A^{\prime}$ точках. Тогда имеем

$$
\nu_{2}+\nu_{3}=\left.D^{3}\right|_{E_{3}} \circ T=D^{3} \circ T \geqslant 2 \nu_{4}+\mu .
$$

Далее, кривая $B_{2}$ не содержится ни в $E_{1}^{2}$, ни в $S^{2}$ и проходит через точку $A$, обшую для $E_{1}^{2}$ и $S^{2}$, поэтому $E_{1}^{3}$ и $S^{3}$ высекают на $E_{3}$ один и тот же слой, так что $A^{\prime} \in E_{1}^{3}$. Таким образом, через точки $B_{1}, A$ и $A^{\prime}$, рассматриваемые как (бесконечно близкие) точки на $E_{1}$, можно провести неприводимую конику, и из пересечения общей такой коники с $D^{1}$ находим, что

$$
2 \nu_{1} \geqslant \nu_{2}+\nu_{3}+\mu
$$

Это неравенство и полученное вьше неравенство $\nu_{2}+\nu_{3} \geqslant 2 \nu_{4}+\mu$ дают третье необходимое нам неравенство $\nu_{1} \geqslant \nu_{4}+\mu$.

Поскольку $\nu_{1}+m_{1}=2 \mu+\alpha_{0} \leqslant \frac{n}{2}+\frac{3}{2} \mu$, то $\nu_{4}+m_{1} \leqslant \frac{n}{2}+\frac{\mu}{2} \leqslant \frac{3}{4} n$, откуда $m_{1}<\frac{n}{4}$ ввиду того, что $\nu_{4}>\frac{n}{2}$. Далее,

$$
\frac{1}{2}(n-\mu) \geqslant \alpha_{0}=e_{1} \circ C^{1} \geqslant \alpha_{1}+\alpha_{2}+\alpha_{3},
$$

так что $\alpha_{3} \leqslant \frac{1}{6}(n-\mu)$. Кроме того, $2 \alpha_{0}+\alpha_{1}+\alpha_{2} \leqslant C \circ l_{0}=n-\mu$. Наконец, $\widetilde{\nu}_{4}=\alpha_{3}+m_{1}+m_{3}$, и мы имеем

$$
\begin{gathered}
2 \nu_{1}+\nu_{2}+\nu_{3}+\nu_{4} \leqslant 6 \mu+2 \alpha_{0}+\alpha_{1}+\alpha_{2}+\alpha_{3}+m_{1} \leqslant 6 \mu+\frac{7}{6}(n-\mu)+m_{1} . \\
\text { Учитьвая, что } 2 \nu_{1} \geqslant \nu_{2}+\nu_{3}+\mu \text { и } \nu_{2}+\nu_{3} \geqslant 2 \nu_{4}+\mu \geqslant \nu_{4}+\nu_{5}+\mu, \text { находим } \\
2 \nu_{1}+\nu_{2}+\nu_{3}+\nu_{4} \geqslant 4 \beta_{4}+2 \mu+\nu_{4},
\end{gathered}
$$

где согласно обозначениям, введенным выше, $\beta_{4}=\frac{1}{4}\left(\nu_{2}+\nu_{3}+\nu_{4}+\nu_{5}\right)$. Тогда имеем

$$
4 \beta_{4}+\nu_{4} \leqslant 4 \mu+\frac{7}{6}(n-\mu)+m_{1}<\frac{7}{6} n+\frac{17}{6} \mu+\frac{n}{4} \leqslant \frac{17}{6} n .
$$

Однако $\nu_{4}>\frac{n}{2}$, и тогда $\beta_{4}<\frac{7}{12} n$, но ранее уже показывалось, что $\beta_{4}>\frac{5}{8} n$ при $N=5$ и $\beta_{6}>\frac{7}{12} n$ при $N=7$. Таким образом, ситуация $B_{3} \cap e_{1}^{3} \neq \varnothing$ также невозможна. 
При $L=2$ осталось рассмотреть последний случай, когда $B_{3} \cap l_{0}^{3}=\varnothing$ и $B_{3} \cap$ $e_{1}^{3}=\varnothing$. Кроме того, $B_{3} \cap E_{2}^{3}=\varnothing$ по свойству конфигурации центров раздутий и исключительных дивизоров, отмеченному в конце $\S 2$. Имеем $\frac{1}{2}(n-\mu) \geqslant \alpha_{0} \geqslant \alpha_{1}+$ $\alpha_{2} \geqslant 2 \alpha_{3}$, т. е. $\alpha_{3} \leqslant \frac{1}{4}(n-\mu)$. Далее, $2 \alpha_{0}+\alpha_{1}+\alpha_{2} \leqslant n-\mu$, и поскольку $\widetilde{\nu}_{4}=\alpha_{3}+m_{3}$ в рассматриваемом случае, то

$$
2 \nu_{1}+\nu_{2}+\nu_{3}+\nu_{4} \leqslant 6 \mu+2 \alpha_{0}+\alpha_{1}+\alpha_{2}+\alpha_{3} \leqslant 6 \mu+\frac{5}{4}(n-\mu) .
$$

Рассуждая так же, как в предыдущем случае, можно получить неравенства $2 \nu_{1} \geqslant \nu_{2}+\nu_{3}+\mu$ и $\nu_{2}+\nu_{3} \geqslant 2 \nu_{4}+\mu$, так что остается верным условие

$$
2 \nu_{1}+\nu_{2}+\nu_{3}+\nu_{4} \geqslant 4 \beta_{4}+2 \mu+\nu_{4}
$$

Таким образом,

$$
4 \beta_{4}+\nu_{4} \leqslant 4 \mu+\frac{5}{4}(n-\mu) \leqslant \frac{21}{8} n,
$$

и поскольку $\nu_{4}>\frac{n}{2}$, то $\beta_{4}<\frac{17}{32} n$, что, как и прежде, противоречит оценкам снизу на $\beta_{i}$ при $N=5$ и $N=7$.

Итак, случай $L=2$ исключен полностью.

Для завершения доказательства утверждения 2), б) теоремы 3.1 остается показать, что ситуации $L=1, N>2$ не реализуются. Как и ранее, пусть $e_{1}=E_{1} \cap S^{1}$, и положим $A=l_{0}^{1} \cap E_{1}$. Заметим, что $l_{0}^{1}$ касается $E_{1}$ в точке $A$. Наши рассуждения зависят от взаимного расположения кривых $B_{1}$ и $e_{1}$ и точки $A$.

Сначала предположим, что $A \in B_{1}$ и $B_{1} \neq e_{1}$. Легко видеть, что после раздутия $B_{1}$ собственный прообраз общей прямой на $E_{1}$, проходящей через точку $A$, будет пересекать собственный прообраз $l_{0}^{1}$, откуда следует, что $\nu_{1} \geqslant \nu_{2}+\mu$. По замечанию, сделанному в конце $\S 2, B_{2} \cap E_{1}^{2}=\varnothing$, так что

$$
\begin{aligned}
& \widetilde{\nu}_{1}=2 \mu+\alpha_{0}, \\
& \widetilde{\nu}_{2}=\mu+\alpha_{1}+m_{1}, \\
& \widetilde{\nu}_{3}=\alpha_{2}+m_{2} .
\end{aligned}
$$

Таким образом,

$$
\nu_{1}+\nu_{2}+\nu_{3} \leqslant 3 \mu+\alpha_{0}+\alpha_{1}+\alpha_{2} \leqslant 3 \mu+n-\mu=n+2 \mu .
$$

Поскольку $\nu_{1} \geqslant \nu_{2}+\mu$, то $2 \nu_{2}+\nu_{3} \leqslant n+\mu \leqslant \frac{3}{2} n$, и мы получаем

$$
\nu_{3} \leqslant \frac{1}{3}\left(2 \nu_{2}+\nu_{3}\right) \leqslant \frac{n}{2} .
$$

Противоречие.

Теперь предположим, что $B_{1}=e_{1}$. Так как $S$ выбрана общей, то $m_{1}=\nu_{2}$. Кроме того, используя те же аргументы, что и вьше, получим, что $\nu_{1} \geqslant \nu_{2}+\mu$. Поскольку $\nu_{1}+m_{1}=2 \mu+\alpha_{0}$, т. е. $\nu_{1}+\nu_{2}=2 \mu+\alpha_{0}$, находим, что $2 \nu_{2} \leqslant \mu+\alpha_{0}$. С другой стороны, $\alpha_{0} \leqslant \frac{1}{2}(n-\mu)$. В итоге $2 \nu_{2} \leqslant \frac{1}{2}(n+\mu)$, откуда $\nu_{2} \leqslant \frac{3}{8} n<\frac{n}{2}$, что невозможно. 
Наконец, пусть $A \notin B_{1}$. Тогда

$$
\begin{aligned}
& \widetilde{\nu}_{1}=2 \mu+\alpha_{0}, \\
& \widetilde{\nu}_{2}=\alpha_{1}+m_{1},
\end{aligned}
$$

откуда $\nu_{1}+\nu_{2} \leqslant 2 \mu+\alpha_{0}+\alpha_{1} \leqslant 2 \mu+n-\mu=n+\mu$. Из пересечения $D$ и общей прямой на $E_{1}$, проходящей через точку $A$, легко вывести, что $\nu_{1} \geqslant \nu_{2}+\mu$, и мы получаем противоречие: $2 \nu_{2} \leqslant n$. Из всего сказанного выше следует, что случай $L=1, N=2$ возможен только тогда, когда $A \in B_{1} \neq e_{1}$.

Итак, утверждение 2), б), а вместе с ним и вся теорема 3.1 доказаны.

\section{§6. Заключительные комментарии}

В настоящей статье было показано, что линейные системы на $V$ могут иметь максимальные особенности либо вдоль кривых семейства $\mathscr{L}$ (линейная система $|H-C|, C \in \mathscr{L}$, дает такой пример), либо над точками типов, перечисленных в пп. 2), а) и 2), б) теоремы 3.1.

Теперь ответим на вопрос: что же нужно, чтобы доказать гипотезу 1.1? Во-первых, либо исключить пп. 2), а) и 2), б) теоремы 3.1 , либо предъявить соответствующие бирациональные автоморфизмы $V$, иначе гипотеза неверна и $V$ содержит какие-то дополнительные структуры Мори. Затем, предполагая, что $\mathscr{D} \subset|n H|$ имеет максимальную особенность вдоль $C \in \mathscr{D}$ с кратностью $\mu>\frac{n}{2}$, мы должны рассмотреть расслоение Мори $V_{C} \rightarrow \mathbb{P}^{1}$, описанное в $\S 1$, и показать, что собственньй прообраз $\mathscr{D}_{V_{C}}$ системы $\mathscr{D}$ на $V_{C}$ (а это подсистема в $\left|(n-\mu)\left(-K_{V_{C}}\right)+(2 \mu-n) F\right|$, где $F$ - класс слоя $\left.V_{C} \rightarrow \mathbb{P}^{1}\right)$ не имеет сверхмаксимальных особенностей. В случае, когда $C$ неособа (а значит, и $V_{C}$ неособо), это было доказано в [1].

Предположим, что $V_{C}$ имеет особую точку. Из рассуждений, приведенных в [1], нетрудно вывести, что необходимо рассмотреть лишь случаи сверхмаксимальных особенностей над точками, лежащими над множеством особенностей специального дивизора $G_{V} \sim\left(-K_{V_{C}}-2 F\right)$, который расслоен над $\mathbb{P}^{1}$ на кривые, изоморфные $C$. Действительно, исключение сверхмаксимальных особенностей надгладкой частью $V_{C}$ не должно вызвать серьезных затруднений. Для исключения максимальных особенностей над двойными точками $V_{C}$ можно использовать следующий результат [9], [5].

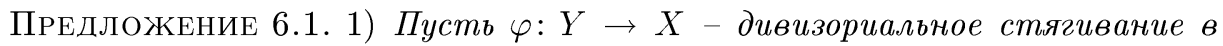
категории Мори в особую точку вида $x y+z w=0$. Тогда $\varphi$ является взвешенным раздутием с весами $(1,1,1,1)$, т.е. $\varphi$ - обыкновенное вложенное раздутие.

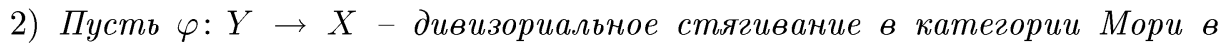
особую точку вида $x y+z^{2}+w^{3}=0$. Тогда $\varphi$ является взвешенным раздутием с весами либо $(1,1,1,1)$, либо $(1,5,3,2)$.

Случай раздутия с весами $(1,1,1,1)$ исключается весьма просто. С другой стороны, весьма проблематичным выглядит исключение максимальных особенностей, реализуемых раздутием с весами $(1,5,3,2)$. Отметим также, что принцип замены максимальных особенностей дивизориальными раздутиями (см. § 2 ) может не быть верным для сверхмаксимальных особенностей. 


\section{Список литературы}

1. Гриненко M. М. Бирациональные свойства пучков поверхностей дель Пеццо степеней 1 и 2 // Матем. сб. 2000. Т. 191. № 5. С. 17-38.

2. Гриненко М. М. О расслоениях на поверхности дель Пеццо // Матем. заметки. 2001. T. 69. № 4. C. 550-565.

3. Исковских В. А. Бирациональные автоморфизмы трехмерных алгебраических многообразий // Итоги науки и техн. Совр. пробл. матем. Т. 12. М: ВИНИТИ, 1979. С. 159-236.

4. Пухликов A. В. Бирациональные автоморфизмы трехмерных алгебраических многообразий с пучком поверхностей дель Пеццо // Изв. РАН. Сер. матем. 1998. Т. 62. № 1. C. $123-164$.

5. Федоров И. Ю. Дивизориальные стягивания в трехмерные $c D v$ точки // Матем. сб. 2002. Т. 193. № 7. С. $149-160$.

6. Хашин С.И. Бирациональные автоморфизмы двойного конуса Веронезе размерности три // Вестн. МГУ. Сер. 1. Математика. Механика. 1984. № 1. С. 13-16.

7. Corti A. Factoring birational maps of threefolds after Sarkisov // J. Algebraic Geom. 1995. V. 4. P. 223-254.

8. Kawakita $M$. Divisorial contractions in dimension 3 which contract divisors to smooth points // Invent. Math. 2001. V. 145. № 1. P. 105-119.

9. Kawakita $M$. Divisorial contractions in dimension 3 which contract divisors to compound $A_{1}$ points // Compos. Math. 2002. V. 133. № 1. P. 95-116.

10. Pukhlikov A. V. Essentials of the method of maximal singularities // Explicit birational geometry of 3-folds. London Math. Soc. Lect. Note Ser. V. 281. Cambridge: Cambridge Univ. Press, 1999. P. 73-100.

Математический институт им. В. А. Стеклова РАН

E-mail: grin@mi.ras.ru

Поступило в редакцию

21.I.2003 